PROCEEDINGS OF THE

AMERICAN MATHEMATICAL SOCIETY

Volume 131, Number 4, Pages 1131-1136

S 0002-9939(02)06699-6

Article electronically published on July 26, 2002

\title{
A LOCAL GEOMETRIC CHARACTERIZATION OF THE BOCHNER-MARTINELLI KERNEL
}

\author{
MICHAEL BOLT
}

(Communicated by Mei-Chi Shaw)

\begin{abstract}
In this paper it is shown that a connected smooth local hypersurface in $\mathbb{C}^{n}$ for which the skew-hermitian part of the Bochner-Martinelli kernel has a weak singularity must lie on a surface having one of the following forms: $S^{2 m+1} \times \mathbb{C}^{n-m-1}$ for some $1 \leq m<n$, or $C \times \mathbb{C}^{n-1}$ where $C$ is a one-dimensional curve. This strengthens results of Boas about the BochnerMartinelli kernel and it generalizes a result of Kerzman and Stein about the Cauchy kernel.
\end{abstract}

\section{INTRODUCTION}

For a smooth hypersurface $M$ in $\mathbb{C}^{n}$ write $\nu_{w}$ for the unit normal at $w$. (If $M$ is the boundary of a domain, then $\nu$ should point outward.) We make the identification of $\mathbb{C}^{n}$ with $\mathbb{R}^{2 n}$ so that a point $w=\left(w_{1}, \ldots, w_{n}\right) \in \mathbb{C}^{n}$ corresponds to $\left(x_{1}, \ldots, x_{2 n}\right) \in \mathbb{R}^{2 n}$ where $w_{j}=x_{j}+i x_{j+n}$. In general, the space to which a vector belongs is to be inferred from the context. Then the Bochner-Martinelli kernel is the function

$$
K(z, w)=\frac{1}{c_{n}} \frac{(\bar{w}-\bar{z}) \cdot \nu_{w}}{|w-z|^{2 n}}, w \in M, z \neq w .
$$

Here $c_{n}$ is the area of a unit sphere in $\mathbb{C}^{n}$ and the product is the complex dot product: $z \cdot w=\sum z_{j} w_{j}$ for $z, w \in \mathbb{C}^{n} . K(z, w)$ is a generalization of the Cauchy kernel, for if $M$ is the boundary of a domain $\Omega$ and $f \in O(\Omega) \cap C(\bar{\Omega})$, then $K f(z)=$ $f(z)$ where, as an operator, $K$ is defined by integration against its kernel,

$$
K f(z)=\int_{b \Omega} K(z, w) f(w) d \sigma_{w}, z \in \Omega
$$

Here $d \sigma$ is Euclidean surface measure. In one dimension, $K(z, w)$ is holomorphic in the $z$ variable and in fact equals the Cauchy kernel. For higher dimensions, however, $K(z, w)$ is not holomorphic.

In [6], Kerzman and Stein showed that if $K(z, w)$ is the Cauchy kernel, then its skew-hermitian part $A(z, w)=K(z, w)-\bar{K}(w, z)$ has a strictly weaker singularity at the boundary diagonal, so $A(z, w)$ is smooth. This means that the operator $A$, which has kernel $A(z, w)$, is compact on $L^{2}(b \Omega)$, so $A$ and $K$ can together be used to represent the Szego" projector via $S=K(I+A)^{-1}$. (See Bell's book [1] for a nice treatment of one-variable complex analysis based on this idea.) Kerzman and

Received by the editors November 1, 2001.

2000 Mathematics Subject Classification. Primary 32A26; Secondary 53A07.

(C)2002 American Mathematical Society 
Stein showed also that $A(z, w)$ vanishes identically precisely when $b \Omega$ is a circle or a line.

In 2], Boas generalized this last result to the Bochner-Martinelli kernel in higher dimensions, thereby answering a question of Kerzman from [5]. Boas showed that $K(z, w) \equiv \bar{K}(w, z)$ in the boundary of a smooth bounded domain precisely when that boundary is a sphere. Later he extended the result in 3 to give a local characterization of hypersurfaces for which $K(z, w) \equiv \bar{K}(w, z)$. Namely, $K(z, w) \equiv$ $\bar{K}(w, z)$ for all $w, z$ in a hypersurface if and only if that hypersurface lies on a surface of the form $S^{2 m+1} \times \mathbb{C}^{n-m-1}$ where $0 \leq m<n$. In [8], Wegner gave a differential geometric proof of the same result.

In this paper we weaken the assumption on the Bochner-Martinelli kernel but recover essentially the same geometric condition for the hypersurface. Notice that $K(z, w)$ has a singularity like $|w-z|^{1-2 n}$ at the diagonal in $M \times M$. Its skewhermitian part,

$$
A(z, w)=\frac{1}{c_{n}} \frac{(\bar{w}-\bar{z}) \cdot \nu_{w}-(z-w) \cdot \bar{\nu}_{z}}{|w-z|^{2 n}}
$$

has a weaker singularity, since rewriting the numerator gives

$$
\begin{aligned}
(\bar{w}-\bar{z}) \cdot \nu_{w}- & (z-w) \cdot \bar{\nu}_{z} \\
& =-2 \operatorname{Re}\left[(z-w) \cdot \bar{\nu}_{w}\right]+(z-w) \cdot\left[\bar{\nu}_{w}-\bar{\nu}_{z}\right] .
\end{aligned}
$$

Then the second term on the right side vanishes to second order at the diagonal, and so does the first term since it is a multiple of the Euclidean inner product of $z-w$ with the normal $\nu_{w} 1$ So if $|a(z, w)| \lesssim|w-z|^{j}$ means there is a $k \in \mathbb{R}$ so that $|a(z, w)| \leq k|w-z|^{j}$ for $z \neq w$, then it follows generally that $|A(z, w)| \lesssim|w-z|^{2-2 n}$. Our result is the following.

Theorem. A connected smooth (local) hypersurface $M \subset \mathbb{C}^{n}$ satisfies $|A(z, w)| \lesssim$ $|w-z|^{3-2 n}$ at each $w \in M(z \neq w)$ if and only if it lies on a surface of the form $S^{2 m+1} \times \mathbb{C}^{n-m-1}$ for some $1 \leq m<n$, or of the form $C \times \mathbb{C}^{n-1}$ where $C$ is a one-dimensional curve.

It is easy to check that the condition on $A(z, w)$ is satisfied for the two kinds of surfaces, so one direction is trivial. The first kind of surface is the kind for which $A(z, w) \equiv 0$, and the second comes from the fact that if $n=1$, then there is always the extra cancelation of singularities, so $A(z, w)$ is bounded and it even vanishes at the diagonal. This appeared in $[6]$ as a remark by Robert Jackson.

We remark that since derivatives of curvatures are involved in the proof of the theorem, $M$ must be assumed at least $C^{3}$ smooth. Boas' method only requires that $M$ be $C^{1}$. For more about the Bochner-Martinelli kernel and its applications, see the book by Kytmanov 7 .

The author thanks Professor Sidney Webster for supervising the work of his doctoral dissertation, of which this was a part, and also the paper's referee for making several helpful comments and pointing out reference [8].

\footnotetext{
${ }^{1}$ If $a=\left(x_{1}, \ldots, x_{2 n}\right)$ and $b=\left(y_{1}, \ldots, y_{2 n}\right)$, then $\operatorname{Re}[a \cdot \bar{b}]=\operatorname{Re} \sum_{j=1}^{n}\left(x_{j}+i x_{j+n}\right)\left(y_{j}-i y_{j+n}\right)$ $=\sum_{j=1}^{2 n} x_{j} y_{j}$.
} 


\section{THE MAIN SINGULARITY}

Consider first the numerator of $A(z, w)$ as given by (2). Suppose that $z: t=$ $\left(t_{1}, \ldots, t_{2 n-1}\right) \in \mathbb{R}^{2 n-1} \rightarrow z_{t} \in M$ parameterizes $M$, and write

$$
z=z(s) \in M, \nu_{z}=\nu(s) \text { and } w=z(t) \in M, \nu_{w}=\nu(t) .
$$

Using the expansions

$$
z(s)-z(t)=\sum_{j=1}^{2 n-1} \partial_{j} z(t)\left(s_{j}-t_{j}\right)+\frac{1}{2} \sum_{i, j=1}^{2 n-1} \partial_{i j} z(t)\left(s_{j}-t_{j}\right)\left(s_{i}-t_{i}\right)+O(s-t)^{3}
$$

and

$$
\nu(s)-\nu(t)=\sum_{i=1}^{2 n-1} \partial_{i} \nu(t)\left(s_{i}-t_{i}\right)+O(s-t)^{2}
$$

we have

$$
\begin{aligned}
&-2 \operatorname{Re}\left[(z-w) \cdot \bar{\nu}_{w}\right]+(z-w) \cdot\left[\bar{\nu}_{w}-\bar{\nu}_{z}\right] \\
&=-\sum_{i, j=1}^{2 n-1} \operatorname{Re}\left[\partial_{i j} z(t) \cdot \bar{\nu}(t)\right]\left(s_{j}-t_{j}\right)\left(s_{i}-t_{i}\right) \\
& \quad-\sum_{i, j=1}^{2 n-1} \partial_{j} z(t) \cdot \partial_{i} \bar{\nu}(t)\left(s_{j}-t_{j}\right)\left(s_{i}-t_{i}\right)+O(s-t)^{3} \\
&=-\sum_{i, j=1}^{2 n-1} i \operatorname{Im}\left[\partial_{j} z(t) \cdot \partial_{i} \bar{\nu}(t)\right]\left(s_{j}-t_{j}\right)\left(s_{i}-t_{i}\right)+O(s-t)^{3}
\end{aligned}
$$

with the last step coming from

$$
\operatorname{Re}\left[\partial_{i j} z \cdot \bar{\nu}+\partial_{j} z \cdot \partial_{i} \bar{\nu}\right]=\partial_{i} \operatorname{Re}\left[\partial_{j} z \cdot \bar{\nu}\right]=0 .
$$

By setting $v_{j}=s_{j}-t_{j}$ we then have $|A(z, w)| \lesssim|w-z|^{3-2 n}$ if and only if

$$
\sum_{i, j} \operatorname{Im}\left[v_{j} \partial_{j} z(t) \cdot v_{i} \partial_{i} \bar{\nu}(t)\right]=0 \text { for all }\left(v_{1}, \ldots, v_{2 n-1}\right) \in \mathbb{R}^{2 n-1} .
$$

Next we interpret this condition independently of the choice of coordinates.

\section{Notation}

We use the following notation, much of which can be found in the book by Hicks [4], for instance. As before, let $w_{1}, \ldots, w_{n}$ be coordinates in $\mathbb{C}^{n}$ with $w_{j}=$ $x_{j}+i x_{j+n}$, so that corresponding real coordinates in $\mathbb{R}^{2 n}$ are $x_{1}, \ldots, x_{2 n}$. The complex structure $J: T \mathbb{R}^{2 n} \rightarrow T \mathbb{R}^{2 n}$ corresponds to multiplication by $\sqrt{-1}$ in $\mathbb{C}^{n}$ and is given by $J\left(\partial_{x_{j}}\right)=\partial_{x_{j+n}}, J\left(\partial_{x_{j+n}}\right)=-\partial_{x_{j}}$. Then $J$ preserves the Euclidean inner product $\langle\cdot, \cdot\rangle$ and $J^{2}=-I, J^{*}=-J$. The complex tangent space of $M$ is $H M=T M \cap J(T M)$. Let $N$ be a unit normal vector on $M$; then the direction orthogonal to $H M$ in $T M$ is $J N$. If $Y$ is any vector field on $M$ and $X \in T M$, then $d_{X} Y$ is the derivative of $Y$ in the $X$ direction. So $d_{X} Y$ is a vector field that might not be tangent to $M$ even if $Y \in T M$. It is not hard to check that $d$ and $J$ commute. 
The map $L: T M \rightarrow T M$ defined by $L(X)=d_{X} N$ is called the Weingarten map. Its image lies in $T M$ for if $X \in T M$, then

$$
0=X\langle N, N\rangle=2\langle L(X), N\rangle .
$$

For $X, Y \in T M$ one has $\langle L(X), Y\rangle=\langle X, L(Y)\rangle$. The (orthogonal) eigenvectors of $L$ are the principal directions of $M$ and the corresponding eigenvalues are the principal curvatures. The relationship between the induced metric on $M \subset \mathbb{R}^{2 n}$ and its curvature form is given in the Codazzi equation

$$
\left\langle d_{X} L(Y)-d_{Y} L(X), Z\right\rangle=\langle L[X, Y], Z\rangle,
$$

valid for $X, Y, Z \in T M$. The brackets indicate taking the commutator, $[X, Y]=$ $d_{X} Y-d_{Y} X$.

So consider again the sum $\sum v_{j} \partial_{j} z(t)$ from condition $\left(\mathrm{C}_{1}\right)$. If $\left(v_{1}, \ldots, v_{2 n-1}\right)$ ranges over $\mathbb{R}^{2 n-1}$, then this sum ranges over $X \in T_{p} M$ where $p=z(t)$. Moreover, the sum $\sum v_{i} \partial_{i} \bar{\nu}(t)$ is $d_{X} \bar{N}$. So $\left(\mathrm{C}_{1}\right)$ can be rewritten

$$
\operatorname{Im}\left(X \cdot d_{X} \bar{N}\right)=0 \text { for every } X \in T_{p} M,
$$

and this is equivalent to

$$
\left\langle X, J d_{X} N\right\rangle=0 \text { for every } X \in T_{p} M
$$

since $\operatorname{Im}(X \cdot \bar{Y})=\langle X, J Y\rangle$ for all $X, Y$. (On the left side of this equation, $X$ and $Y$ are viewed as vectors in $\mathbb{C}^{n}$ with the complex dot product; on the right side they are in $\mathbb{R}^{2 n}$ with the Euclidean product.) In what remains we assume that $\left(\mathrm{C}_{2}\right)$ is satisfied at all $p \in M$. This is the hypothesis of the Theorem.

\section{Proof of the Theorem}

The main idea, as in [8], is to show that there are at most two distinct values for the principal curvatures at different points of the surface, and if there are two, then one of them must be equal to zero.

Lemma 1. For all $X, Y \in T_{p} M,\left\langle X, J d_{Y} N\right\rangle+\left\langle Y, J d_{X} N\right\rangle=0$.

Proof. Since also $X+Y \in T_{p} M$,

$$
\begin{aligned}
0=\left\langle X+Y, J d_{X+Y} N\right\rangle & =\left\langle X, J d_{X} N\right\rangle+\left\langle X, J d_{Y} N\right\rangle \\
+ & \left\langle Y, J d_{X} N\right\rangle+\left\langle Y, J d_{Y} N\right\rangle=\left\langle X, J d_{Y} N\right\rangle+\left\langle Y, J d_{X} N\right\rangle .
\end{aligned}
$$

Lemma 2. $J N \in T_{p} M$ is a principal direction. The remaining principal directions span $H_{p} M$.

Proof. Since $d_{J N} N \in T_{p} M$ we need only show $d_{J N} N$ is orthogonal to the complex tangent space. So take $X \in H_{p} M$ and apply Lemma 1 to $J X, J N \in T_{p} M$. Then

$$
\begin{aligned}
0=\left\langle J X, J d_{J N} N\right\rangle+ & \left\langle J N, J d_{J X} N\right\rangle \\
& =\left\langle X, d_{J N} N\right\rangle+\left\langle N, d_{J X} N\right\rangle=\left\langle X, d_{J N} N\right\rangle .
\end{aligned}
$$

Lemma 3. Suppose $X \in H_{p} M$ is a principal direction associated to $k^{X}$. Then $J X$ is another principal direction associated to $k^{X}$. 
Proof. Note that $d_{J X} N$ is orthogonal to $J N$ since $J X$ can be written as a combination of the principal directions which span $H_{p} M$.

Now let $Y$ range over the principal directions in $H_{p} M$, so the set of $J Y$ gives a basis for $H_{p} M$. Applying the first lemma to $J X, Y \in T_{p} M$ we have

$$
\begin{array}{r}
0=\left\langle J X, J d_{Y} N\right\rangle+\left\langle Y, J d_{J X} N\right\rangle=\left\langle X, d_{Y} N\right\rangle-\left\langle J Y, d_{J X} N\right\rangle \\
=k^{Y}\langle X, Y\rangle-\left\langle J Y, d_{J X} N\right\rangle .
\end{array}
$$

If $Y \neq X$ this gives $\left\langle J Y, d_{J X} N\right\rangle=0$, and if $Y=X$, then $\left\langle J X, d_{J X} N\right\rangle=k^{X}$. Since the $J Y$ form a basis for $H_{p} M$ it follows that $d_{J X} N=k^{X} J X$.

Lemma 4. Suppose $X$ is a principal direction in the complex tangent space associated to $k^{X}$. Then $k^{X}=k^{J N}$ or $k^{X}=0$.

Proof. We apply the Codazzi equation to $X, J N$, and $J X \in T_{p} M$,

$$
\left\langle d_{X} L(J N)-d_{J N} L(X), J X\right\rangle=\left\langle L\left(d_{X} J N-d_{J N} X\right), J X\right\rangle .
$$

Then on the left side we have

$$
\begin{gathered}
\left\langle d_{X}\left(k^{J N} J N\right), J X\right\rangle-\left\langle d_{J N}\left(k^{X} X\right), J X\right\rangle \\
=X\left(k^{J N}\right)\langle J N, J X\rangle+k^{J N}\left\langle d_{X} J N, J X\right\rangle \\
\quad-(J N)\left(k^{X}\right)\langle X, J X\rangle-k^{X}\left\langle d_{J N} X, J X\right\rangle \\
=k^{J N}\left\langle d_{X} J N, J X\right\rangle-k^{X}\left\langle d_{J N} X, J X\right\rangle,
\end{gathered}
$$

and on the right we have

$$
\begin{aligned}
\left\langle d_{X} J N-d_{J N} X, L(J X)\right\rangle= & \left\langle d_{X} J N, k^{X} J X\right\rangle-\left\langle d_{J N} X, k^{X} J X\right\rangle \\
& =k^{X}\left\langle d_{X} J N, J X\right\rangle-k^{X}\left\langle d_{J N} X, J X\right\rangle .
\end{aligned}
$$

It follows that

$$
\begin{aligned}
0=\left(k^{J N}-k^{X}\right)\left\langle d_{X} J N, J X\right\rangle & =\left(k^{J N}-k^{X}\right)\left\langle J d_{X} N, J X\right\rangle \\
& =\left(k^{J N}-k^{X}\right)\left\langle d_{X} N, X\right\rangle=\left(k^{J N}-k^{X}\right) k^{X} .
\end{aligned}
$$

Following the last two lemmas we denote orthonormal principal directions corresponding to curvature $k=k^{J N}$ by $X_{0}, \ldots, X_{2 m}$, with $X_{0}=J N$, and the orthonormal principal directions corresponding to curvature zero by $Y_{2 m+1}, \ldots, Y_{2 n-2}$.

Lemma 5. $Y_{i}(k)=0$ for all $i$.

Proof. Apply the Codazzi equation to $J N, Y_{i}$, and $J N$,

$$
\left\langle d_{J N} L\left(Y_{i}\right)-d_{Y_{i}} L(J N), J N\right\rangle=\left\langle L\left(d_{J N} Y_{i}-d_{Y_{i}} J N\right), J N\right\rangle .
$$

Then on the left side we have

$$
-\left\langle d_{Y_{i}}(k J N), J N\right\rangle=-Y_{i}(k)\langle J N, J N\rangle-k\left\langle d_{Y_{i}} J N, J N\right\rangle=-Y_{i}(k),
$$

and on the right we have

$$
\begin{aligned}
& \left\langle d_{J N} Y_{i}-d_{Y_{i}} J N, k J N\right\rangle \\
& \quad=k\left\langle d_{J N} Y_{i}, J N\right\rangle-k\left\langle d_{Y_{i}} J N, J N\right\rangle=k\left\langle d_{J N} Y_{i}, J N\right\rangle .
\end{aligned}
$$

Now using Lemma 1 with $J N$ and $Y_{i}$ we have

$$
0=\left\langle J N, J d_{Y_{i}} N\right\rangle+\left\langle Y_{i}, J d_{J N} N\right\rangle=\left\langle Y_{i}, d_{J N} J N\right\rangle .
$$

It follows that $Y_{i}(k)=-k\left\langle d_{J N} Y_{i}, J N\right\rangle=k\left\langle Y_{i}, d_{J N} J N\right\rangle=0$. 
Lemma 6. If $m \neq 0$, then $X_{i}(k)=0$ for all $i$.

Proof. For $i \neq j$, apply the Codazzi equation to $X_{i}, X_{j}$, and $X_{j}$,

$$
\left\langle d_{X_{i}} L\left(X_{j}\right)-d_{X_{j}} L\left(X_{i}\right), X_{j}\right\rangle=\left\langle L\left(d_{X_{i}} X_{j}-d_{X_{j}} X_{i}\right), X_{j}\right\rangle .
$$

Then on the left side we have

$$
\begin{aligned}
\left\langle d_{X_{i}}\left(k X_{j}\right), X_{j}\right\rangle & -\left\langle d_{X_{j}}\left(k X_{i}\right), X_{j}\right\rangle=X_{i}(k)\left\langle X_{j}, X_{j}\right\rangle+k\left\langle d_{X_{i}} X_{j}, X_{j}\right\rangle \\
& -X_{j}(k)\left\langle X_{i}, X_{j}\right\rangle-k\left\langle d_{X_{j}} X_{i}, X_{j}\right\rangle=X_{i}(k)-k\left\langle d_{X_{j}} X_{i}, X_{j}\right\rangle,
\end{aligned}
$$

and on the right we have

$$
\begin{aligned}
\left\langle d_{X_{i}} X_{j}-d_{X_{j}} X_{i}, k X_{j}\right\rangle & \\
& =k\left\langle d_{X_{i}} X_{j}, X_{j}\right\rangle-k\left\langle d_{X_{j}} X_{i}, X_{j}\right\rangle=-k\left\langle d_{X_{j}} X_{i}, X_{j}\right\rangle .
\end{aligned}
$$

It follows that $X_{i}(k)=0$.

Proof of the Theorem. If $m \neq 0$, then $k$ is constant and the second fundamental form is completely determined. In particular, $M$ is part of a surface of the form $S^{2 m+1} \times \mathbb{C}^{n-m-1}$, where $S$ is a sphere of radius $k^{-1}$ inside $\mathbb{C}^{m+1} \cong \mathbb{R}^{2 m+2}$. This is the same condition that Boas determined for when $A(z, w)$ vanishes identically.

If $m=0$, then the curvature is arbitrary in the $J N$ direction but is constant in every $Y$ direction. In particular, $M$ is part of a $C \times \mathbb{C}^{n-1}$ where $C$ is a curve in a one-dimensional complex plane.

\section{REFERENCES}

[1] Steven R. Bell. The Cauchy transform, potential theory, and conformal mapping. CRC Press, Boca Raton, FL, 1992. MR 94k:30013

[2] Harold P. Boas. A geometric characterization of the ball and the Bochner-Martinelli kernel. Math. Ann., 248(3):275-278, 1980. MR 81m:32029

[3] Harold P. Boas. Spheres and cylinders: a local geometric characterization. Illinois J. Math., 28(1):120-124, 1984. MR 85i:53005

[4] Noel J. Hicks. Notes on differential geometry. D. Van Nostrand Co., Inc., Princeton, N.J.Toronto-London, 1965. MR 31:3936

[5] N. Kerzman. Singular integrals in complex analysis. In Harmonic analysis in Euclidean spaces (Proc. Sympos. Pure Math., Williams Coll., Williamstown, Mass., 1978), Part 2, pages 3-41. Amer. Math. Soc., Providence, R.I., 1979. MR 80m:32005

[6] N. Kerzman and E. M. Stein. The Cauchy kernel, the Szegö kernel, and the Riemann mapping function. Math. Ann., 236(1):85-93, 1978. MR 58:6199

[7] Alexander M. Kytmanov. The Bochner-Martinelli integral and its applications. Birkhäuser Verlag, Basel, 1995. Translated from the Russian by Harold P. Boas and revised by the author. MR 97f:32004

[8] Bernd Wegner. A differential geometric proof of the local geometric characterization of spheres and cylinders by Boas. Math. Balkanica (N.S.), 2(4):294-295 (1989), 1988. MR 90b:53004

Department of Mathematics, University of Michigan, Ann Arbor, Michigan 48109

E-mail address: mbolt@umich.edu 